\title{
Android based self-diagnostic electrocardiogram system for mobile healthcare
}

\author{
Kan-Yeep Choo, Huo-Chong Ling*, Yew-Chiong Lo, Zuo-Han Yap, Jun-Sheng Pua, \\ Raphael C.-W. Phan and Vik-Tor Goh \\ Faculty of Engineering, Multimedia University, Cyberjaya, Selangor, Malaysia
}

\begin{abstract}
.
BACKGROUND: Cardiovascular diseases are the most common cause of death worldwide and are characterized by arrhythmia (i.e. irregular rhythm of heartbeat). Arrhythmia occasionally happens under certain conditions, such as stress. Therefore, it is difficult to be diagnosed using electrocardiogram (ECG) devices available in hospitals for just a few minutes. Constant diagnosis and monitoring of heartbeat is required to reduce death caused by cardiovascular diseases.

OBJECTIVE: Mobile healthcare system has emerged as a potential solution to assist patients in monitoring their own heart condition, especially those who are isolated from the reference hospital. This paper proposes a self-diagnostic electrocardiogram system for mobile healthcare that has the capability to perform a real-time ECG diagnostic.

METHODS: The self-diagnostic capability of a real-time ECG signal is achieved by implementing a detrended fluctuation analysis (DFA) method. The result obtained from DFA is used to display the patient's health condition on a smartphone anytime and anywhere. If the health condition is critical, the system will alert the patient and his medical practitioner for further diagnosis.

RESULTS: Experimental results verified the validity of the developed ECG diagnostic application on a smartphone.

CONCLUSION: The proposed system can potentially reduce death caused by cardiovascular diseases by alerting the patient possibly undergoing a heart attack.
\end{abstract}

Keywords: Detrended fluctuation analysis, electrocardiogram, mobile healthcare, self-diagnostic, android smartphone

\section{Introduction}

Cardiovascular diseases are the number one cause of death globally. The number of people who die from cardiovascular diseases, mainly from heart attacks and strokes, will increase to reach 23.3 million by 2030 [1]. Cardiovascular diseases are usually characterized by arrhythmia. An arrhythmia is a problem with the rhythm of the heartbeat. During an arrhythmia, the heart can beat too fast, too slow, or with an irregular rhythm. This abnormal heartbeat happens occasionally under certain conditions, such as stress. Therefore, this condition is difficult to be diagnosed using electrocardiogram (ECG) devices that are available in the hospital for just a few minutes. Constant diagnosis and monitoring of the heartbeat is necessary to reduce death caused by cardiovascular diseases.

\footnotetext{
${ }^{*}$ Corresponding author: Huo-Chong Ling, Faculty of Engineering, Multimedia University, Cyberjaya, Selangor 63100, Malaysia. Tel.: +603 83125388; Fax: +603 83183029; E-mail: hcling@mmu.edu.my/linghchong@gmail.com. 
With the rapid development in wearable medical sensors and wireless communication, mobile healthcare systems [2-10] have emerged as a potential solution to assist patients in monitoring their own heart condition instantly anytime and anywhere. Mobile healthcare system is especially helpful to those who is isolated or far from the reference hospital. In the mobile system, small wearable sensor nodes are placed in, on, or around a patient's body (viz. body area network (BAN)), which are capable of sensing, sampling, processing and transmitting data (e.g. heart rate, blood pressure) without constraining the activities of the patient. The gathered data is then forwarded in real time to the hospital, health center, or central repository over a wireless communications channel for further diagnosis.

\subsection{Related work}

Figueredo and Dias [2] proposed a tele-homecare system for patient monitoring using mobile phone. At the client side, the patient was connected to a patient monitor device at home. Vital signals such as ECG, heart rate and blood pressure were acquired and transmitted by the device to the server side through a mobile phone which was connected to an RS232 interface at the monitoring device. At the server side (normally in hospital or clinic), the data was stored in a database, and the patient was monitored by a medical practitioner using a server application.

Kim et al. [3] proposed a ubiquitous ECG monitoring system using SIP and wireless Zigbee network that could be used by a medical practitioner at the remote side for ECG monitoring, analysis and diagnosis of the patient. The medical practitioner examined the received ECG data and then feedbacked to the patient through the Internet telephone. Borromeo et al. [4] developed a low-cost system for ECG acquisition and visualization in mobile devices which could be easily used by any patient. Borromeo's system is only capable of acquiring the ECG signals and visualizing the signals in the mobile device. It does not have any connection to the database at the health care provider for further analysis and diagnosis.

Noureddine and Selma [5] proposed a design of a mobile personal ECG monitoring system with patient location. An ECG signal acquisition circuit was integrated with a module that communicates with smart mobile phone through Bluetooth. Imec and Holst Centre, together with TASS software professionals [6] developed a mobile heart monitoring system that allows viewing ECG signals on an Android mobile phone.

Chan et al. [7] proposed an ECG monitor system based on Android smartphone. ECG signal was transmitted to the Android smartphone and then forwarded to a remote server for a medical practitioner to view the ECG after logging into the server. Guo et al. [8] designed and developed an Android-based ECG monitoring and alarming system for a medical practitioner to monitor the ECG of the patient outside the hospital. In their system, ECG signal was collected and transmitted by an ECG device to an Android phone. Once the alarm was detected, ECG images and the details about the alarm were captured and sent by the Android phone to a cloud alarm server for the medical practitioner to download the ECG images and alarm details on mobile phone for diagnostic.

Sukanesh et al. [9] proposed a system for long term real-time continuous monitoring of cardiac patients through GSM and wireless technology that enhanced the mobility for both the medical practitioner as well as the patient. The system enabled the medical practitioner to be aware of the cardiac functioning of the patient at a distance. Li et al. [10] developed and implemented a ubiquitous healthcare system dedicated to real-time continuous cardiac arrhythmia detection and monitoring under the surveillance of a medical practitioner.

However, none of the ECG system discussed so far has incorporated self-diagnostic capability in the mobile healthcare system, in other words, the ability of the system to determine the health condition 
of a patient based on the analysis done on the ECG signal. Usually, a medical practitioner uses visual inspection to analyse ECG signal of the patient and then determine whether the patient is in good health condition or not. Thus, the experience of a medical practitioner plays a crucial role in interpreting the ECG signal. An inexperienced medical practitioner could have misinterpreted the ECG signal due to wrong judgments on the visual inspection of the ECG signal. Computerised ECG diagnostic had been proposed to avoid the risk of misinterpreting ECG signal and to standardise the analysis procedures [11, 12]. The computerised ECG diagnostic can also greatly reduce the time taken by a medical practitioner to diagnose a huge number of ECG signals in a more efficient way. One of the potential methods that can be explored and used to analyse the ECG signal is the detrended fluctuation analysis (DFA) [13-16].

In this paper, an Android based self-diagnostic mobile healthcare system using DFA method is proposed. The proposed system has the capability of performing real-time ECG acquisition, diagnosis, visualization and storing in both mobile phone and base station. DFA is a scaling analysis method that provides a simple quantitative parameter to represent the autocorrelation properties of a signal, in particularly non-stationary signal [16]. Therefore, it is useful for analyzing ECG signals, which are normally complex and non-stationary. The scaling exponent obtained from the DFA method can be used to distinguish between healthy and unhealthy subject. Hence, using the scaling exponent, patient's health condition can be displayed on the mobile phone anytime and anywhere. If the health condition is critical, the system will alert the patient and his medical practitioner for further diagnosis.

Besides that, the proposed system has low-power consumption and user-friendliness so that the patient can use it at ease without unnecessarily constraining the patient's daily activities. The developed ECG signal analysis module is deployed in an open-source smartphone operating system. Such a choice is made due to its hardware interoperability, versatility, and usability characteristics.

This paper is organized as follows. In Section 2, Detrended Fluctuation Analysis (DFA), a method used to analyse an ECG signal in the proposed system is discussed in details. In Section 3, the design and implementation of the system are presented. The experimental results of the system proposed in Section 3 are then presented in Section 4. Finally, Section 5 concludes this paper.

\section{Detrended Fluctuation Analysis}

DFA is a statistical method that has been developed to study the existence of short-range or long-range correlation of time series. This method has first been used to study DNA sequence [17] and progressively finds its applications in other time series analysis such as ECG analysis [13-16] and electroencephalogram (EEG) analysis [18--20]. This method consists of a few important steps [13/17|20] as follows:

1. The times series $x(i)$ is subtracted from the mean value $\langle x\rangle$ and then integrated as given in Eq. (1).

$$
Y(j)=\sum_{i=1}^{j} x(i)-\langle x\rangle .
$$

2. The integrated time series $Y(j)$ is divided into boxes of equal length $n$.

3. A linear least-squares line is fitted in each box of length $n$ in which it reflects the local trend in that box, and its $y$-coordinate of the local trend is represented by $Y_{f t}(j)$.

4. The integrated time series $Y(j)$ is then detrended by subtracting it from the local trend $Y_{f i t}(j)$ in each box.

5. The root mean square fluctuation $F(n)$ is computed by:

$$
F(n)=\sqrt{\frac{1}{N} \sum_{j=1}^{N}\left[Y(j)-Y_{f i t}(j)\right]^{2}} .
$$

where $N$ is the length of the time series. 


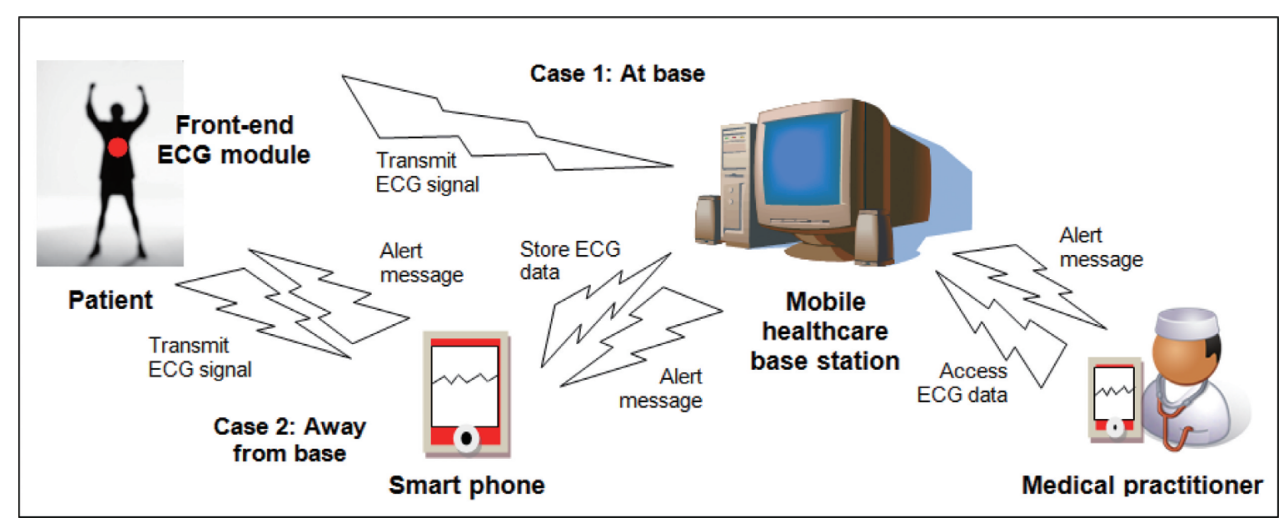

Fig. 1. Proposed Android based self-diagnostic ECG system for mobile healthcare.

6. $F(n)$ is repetitively calculated for all box sizes so that a double logarithm graph could be plotted for $F(n)$ as a function of the box size $n, F(n) \propto n^{\alpha}$. The slope of this double logarithm graph is associated to a scaling exponent $\alpha$ that describes the characteristics of the time series.

For $\alpha=0.5$, the integrated time series are associated to a random walk whose original time series do not present any correlation (white noise) between the data. When $\alpha$ is in between 0.5 and 1.0, the persistent long-range power-law correlations are observed in the time series. In contrast, the anti-persistent long-range power-law correlations are observed in the time series if $\alpha$ is in the range between 0 and 0.5 . If $\alpha=1$, the time series are corresponding to $1 / f$ noise and if $\alpha>1$, the correlation of the time series is not following a power-law form. The integrated time series are considered as a Brown noise (integration of white noise) when $\alpha=1.5$.

In short, the $\alpha$ value is used as a numerical indicator to differentiate various types of cardiovascular diseases. An $\alpha$ less than 1 (for small window size) indicates that the patient may subject to cardiovascular disease and an $\alpha$ greater than 1.0 indicates that the patient is healthy.

\section{Design and implementation}

Figure 1 illustrates the proposed Android based self-diagnostic ECG system for mobile healthcare. Based on Fig. 1, the patient will wear a front-end ECG module on his or her body. The front-end ECG module consists of sensors, ECG signal acquisition and transmission components. The sensors will acquire the ECG signal and transmit the signal through wireless technology (such as Bluetooth or GSM) to a healthcare base station (which acts as a server) or the smartphone if the patient is away from the base station. Both healthcare base station and smartphone are capable of performing ECG signal diagnosis using DFA method, and then display the patient's health condition on the screen. If the condition is critical, the base station will alert the patient and the patient's medical practitioner. The medical practitioner will access the base station to acquire the patient's ECG data for further diagnosis.

The process of acquiring input signal (i.e. heartbeat) from the patient to transmitting ECG signal to the base station and smartphone is performed through the front-end ECG module. The input signals which are generated from the heart are in the form factor of electric potential (i.e. voltage). Thus, three electrodes that function as electric conductors are attached to the human skin surface to transfer electrical heart pulses (i.e. electric potential signals) to the analog front-end (AFE) component of the front-end ECG module. AFE is a set of analog signal circuitry to manipulate input signals for further digital 


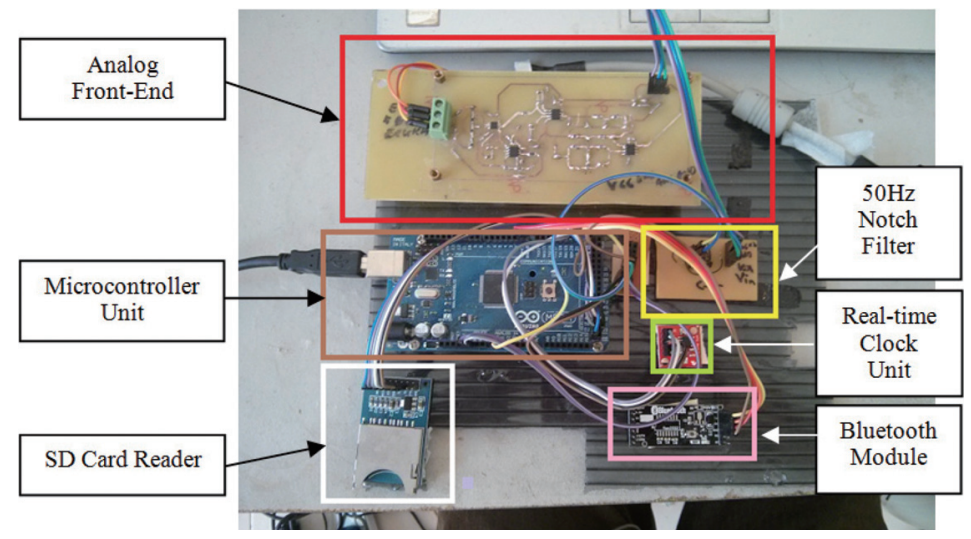

Fig. 2. Prototype of front-end ECG module.

processing. According to the electrical signal of ECG, frequency range of the electrical signal of heart is from $0.1 \mathrm{~Hz}$ to $300 \mathrm{~Hz}$ and amplitude range is from $0.05 \mathrm{mV}$ to $3 \mathrm{mV}$. There are four major amplifiers in the analog front end, namely an instrumentation amplifier, a gain amplifier, a high-pass filter, a low-pass filter, and a notch filter. Instrumentation amplifier is used to amplify the difference between two voltages from the heart. The feedback high-pass and low-pass filters are used to allow signals between $0.1 \mathrm{~Hz}$ and $300 \mathrm{~Hz}$ to pass. An amplifier gain of 500 times is used to increase the amplitude of millivolt ECG signal for digital conversion. A notch filter is designed to block electric power frequency of $50 \mathrm{~Hz}$ or $60 \mathrm{~Hz}$, which is the unwanted noise received in the waveform.

The processed signal is then fed into a microcontroller component, which acts as a central control unit to acquire, manage and manipulate signal. The tasks performed by microcontroller component ranges from converting the analog signal into digital data, storing data into the data storage component, reading data from the data storage component to managing data transfer between components in the front-end ECG module.

In order to have the mobility, wireless communication component provides the module with the capability to remotely link to smartphone or base station. Wired communication component offers an alternative connection if the wireless communication fails. In wireless communication, Bluetooth technology is used to transfer data since Bluetooth is a common and low-power network in most smartphones and computers. USB connection is used for wired communication in the module since the USB is the most common connection for computers, and it can supply power and charge battery for the module.

Data storage component is used to store long-term ECG data recording. Power management component is used to control the charging and discharging of a Li-ion battery that supplies power to the front-end ECG module. It consists of a linear battery charger circuit that provides charging function for the Li-ion battery and a set of linear regulator that supplies constant voltage to the module.

\section{Experimental results}

Figure 2 shows the prototype of the front-end ECG module of the proposed system. The block units shown in the figure include microcontroller development board, circuitry of analog front-end, SD card reader as data storage, Bluetooth module, $50 \mathrm{~Hz}$ Notch filter to filter off unwanted noise in the ECG signal acquisition, and real-time clock unit to provide the current time of the system. 


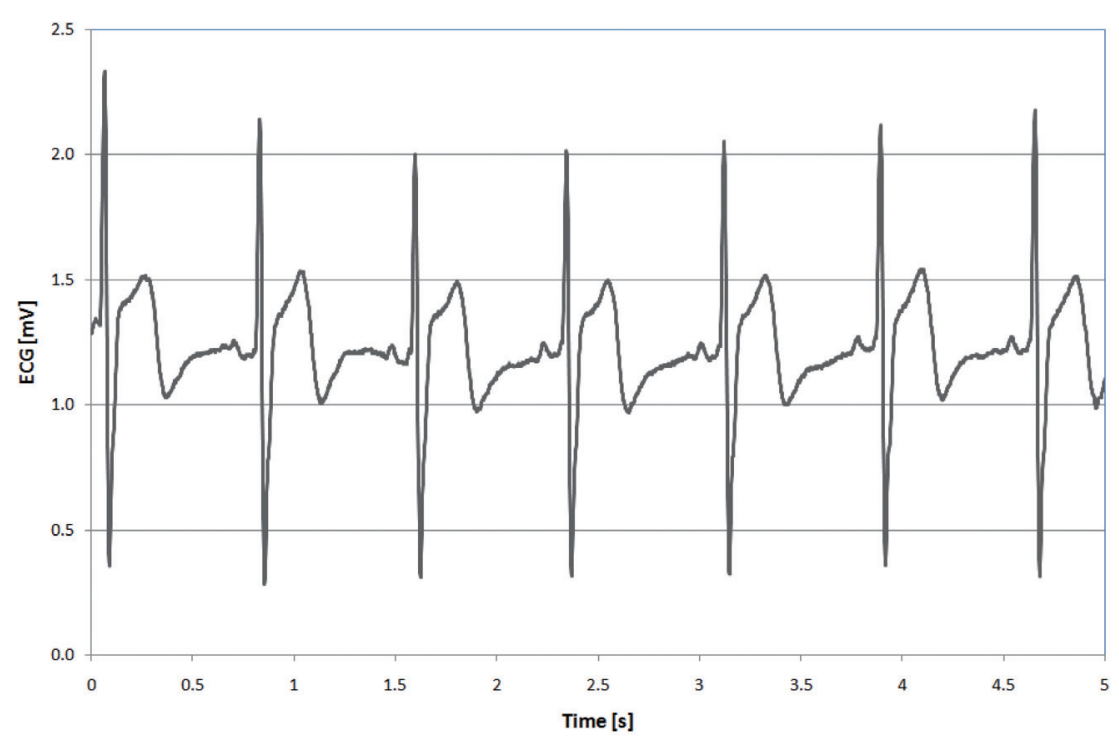

(a)

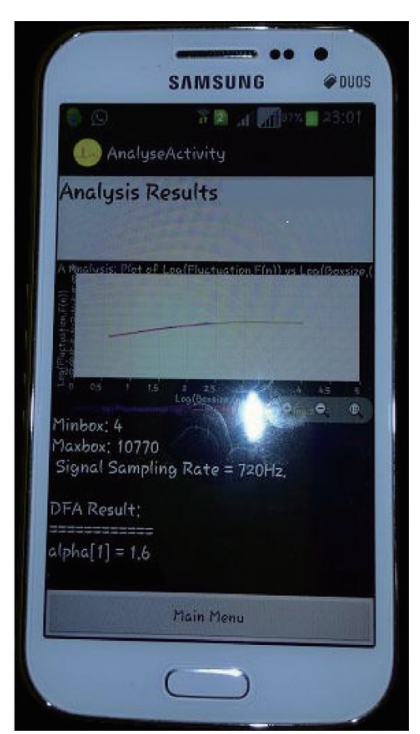

(b)

Fig. 3. (a) Acquired ECG signals after going through the front-end ECG module. (b) Analysis obtained from the ECG diagnostic application installed on an Android-based smartphone.

The signals are captured with three electrodes that are connected to skin surface around the heart. The 3 electrodes are placed on left and right shoulders as well as the pubis. The connection which is called Einthoven's triangle forms a triangle shape and the heart is at the center of the shape. The voltage range is set to between $0 \mathrm{~V}$ to $5 \mathrm{~V}$. Figure 3(a) shows the acquired ECG signals after going through the front-end ECG module. The sampling rate of the ECG signal is $500 \mathrm{~Hz}$. This ECG signal is stored in numeric values in data storage (i.e. SD card). It can also be used for further processing on other platforms, for example, the data can be downloaded by medical practitioner for further diagnostics in case an emergency occurs that requires immediate attention.

The acquired ECG data is then transmitted to smartphone or base station through Bluetooth for further diagnostic of the patient's health using DFA method. An ECG diagnostic application using DFA method has been designed, developed and implemented on an Android based smartphone. Figure 3(b) shows the diagnostic result of one of the ECG data obtained. The $\alpha$ value calculated by the application is 1.6, indicating that the patient is healthy.

In order to verify the validity of the result obtained from the ECG diagnostic application, two ECG signals were used, viz. the standard ECG test signal (aaim3a) obtained from the ANSI/AAMI EC13:2002 [21] and the ECG signal acquired from the front-end ECG module. These two ECG signals were each fed into the ECG diagnostic application and the DFA C code developed in [13|22], respectively to obtain the root mean square fluctuation $F(n)$. A graph as shown in Fig. 4 was then plotted to show the comparison of the $F(n)$ calculated for both signals using the ECG diagnostic application and the DFA code, respectively. The $F(n)$ calculated for the standard ECG test signal (aaim3a) using the DFA C code developed in [13/22] is represented by the black colour line and using the Android code developed in this work is represented by empty black circle. On the other hand, the $F(n)$ calculated for the standard acquired ECG signal in this work using the DFA C code developed in [13/22] is represented by the red colour line and using the Android code developed in this work is represented by empty red square. 


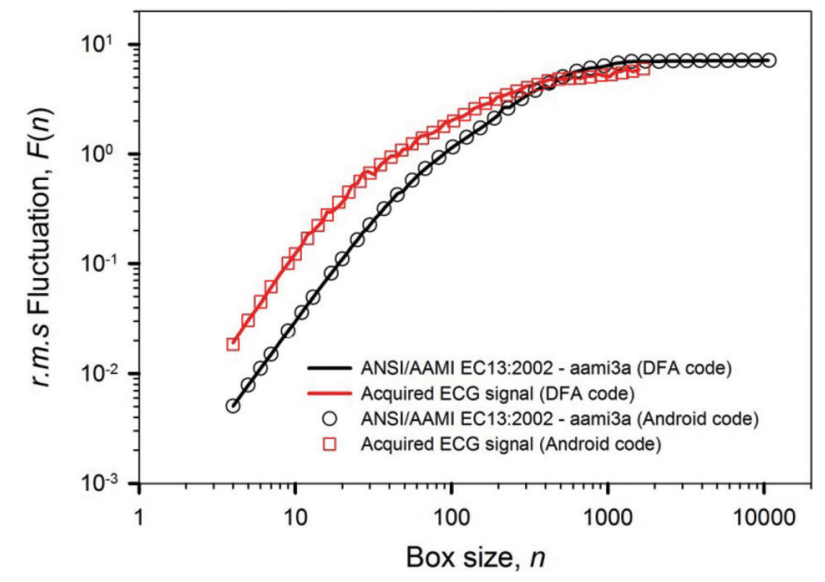

Fig. 4. Comparison of the root mean square fluctuation $F(n)$ calculated for the standard ECG test signal (aaim3a) obtained from the ANSI/AAMI EC13: 2002 [20] and the acquired ECG signal using the ECG module developed in this work.

The graph verified the validity of the result obtained from the ECG diagnostic application as the $F(n)$ calculated from the application aligned perfectly with the $F(n)$ calculated from the DFA code. The $\alpha$ values obtained for the standard ECG test signal (aaim3a) and the acquired signal are 1.6 and 1.5, respectively, indicating that both subjects are healthy.

\section{Conclusions}

This paper proposed a self-diagnostic ECG system for mobile healthcare that can perform diagnosis on the real-time ECG signal acquired from a patient through a front-end ECG module. The front-end ECG module comes with wireless communication for real-time ECG acquisition, diagnosis, visualization and storing in both smartphone and base station. The self-diagnostic capability of real-time ECG signal is achieved by analysing the signal using DFA method in the developed ECG diagnostic application installed on the smartphone of the patient. The result obtained from the application is used to display patient's health condition on the smartphone anytime and anywhere. Experimental results verified the validity of the developed ECG diagnostic application on a smartphone. The idea and the system designed in this paper can also be extended into other electro-diagnostic services, such as electroencephalogram (EEG), electromyogram (EMG) and electromammogram, or any combination of them.

\section{Acknowledgements}

The work presented in this paper was funded by Malaysian Science Fund (01-02-01-SF0189), Ministry of Science, Technology and Innovation (MOSTI) Malaysia through Faculty of Engineering, Multimedia University (MMU).

\section{References}

[1] WHO. Cardiovascular diseases [Internet]. World Health Organization. 2014; [updated 2013 March 27; cited 2014 Jul 11]. Available from: http://www.who.int/cardiovascular_diseases/en/. 
[2] Figueredo MVM, Dias JS. Mobile telemedicine system for home care and patient monitoring. Proceedings of the 26th Annual Int. Conf. IEEE EMBS. 2004; 3387-3390.

[3] Kim B, Kim Y, Lee I, You I. Design and implementation of a ubiquitous ECG monitoring system using SIP and the Zigbee network. Proceedings of FGCN. 2007; 599-604.

[4] Borromeo S, Sanchez CR, Machado F, Tamames JAH, Prieta R. A reconfigurable, wearable, wireless ECG system. Proceedings of the 29th Annual Int Conf IEEE EMBS. 2007; 1659-1662.

[5] Noureddine B, Selma B. Mobile personal electrocardiogram monitoring system with patient location. Proceedings of WiMD. 2009; 69-71.

[6] Imec news. Monitoring your health with your mobile phone [Internet]. Netherlands: Imec; [updated 2010 Oct 5; cited 2014 Jul 11]. Available from: http://www2.imec.be/be_en/press/imec-news/wirelesshealthnecklaceinterface.html.

[7] Chan CC, Chen CW, Chou WC, Ho YL, Lin YH, Ma HP. Live demonstration: A mobile ECG healthcare platform. IEEE Biomedical Circuits and Systems Conference. 2012; 87.

[8] Guo X, Duan X, Gao H, Huang A, Jiao B. An ECG monitoring and alarming system based on android smart phone. Communications and Network. 2013; 5: 584-589.

[9] Sukanesh R, Veluchamy S, Karthikeyan M. A portable wireless ECG monitoring system using GSM technique with real time detection of beat abnormalities. International Journal of Engineering Research. 2014; 3(2): 108-111.

[10] Li J, Zhou H, Zuo D, Hou K, Vaulx CD. Ubiquitous health monitoring and real-time cardiac arrhythmias detection: A case study. Bio-Medical Materials and Engineering. 2014; 24: 1027-1033.

[11] Kalovrektis K, Ganetsos T, Shammas NYA, Taylor I, Andonopoulos J. Development of a novel system to analyse and detect small changes in ECG signals that indicate cardiac disorders. Proceedings of the 4th WSEAS Int Conf on Circuits, Systems, Signal and Telecommunications (CISST '10). 2010; 86-89.

[12] Oweis R, Hijazi L. A computer-aided ECG diagnostic tool. Computer Methods and Programs in Biomedicine. 2006; 81(3): 279-284.

[13] Peng CK, Havlin S, Stanley HE, Goldberger AL. Quantification of scaling exponents and crossover phenomena in nonstationary heartbeat time series. Chaos. 1995; 5(1): 82-87.

[14] Meyer M, Stiedl O. Self-affine fractal variability of human heartbeat interval dynamics in health and disease. Eur J Appl Physiol. 2003; 90: 305-316.

[15] Huikuri HV, Perkiömäki JS, Maestri R, Pinna GD. Clinical impact of evaluation of cardiovascular control by novel methods of heart rate dynamics. Phil Trans R Soc. A. 2009; 367(1892): 1223-1238.

[16] Golińska AK. Detrended fluctuation analysis (DFA) in biomedical signal processing: Selected examples. Studies in Logic, Grammar and Rhetoric. 2012; 29(42): 107-115.

[17] Peng CK, Buldyrev SV, Havlin S, Simons M, Stanley HE, Goldberger AL. On the mosaic organization of DNA nucleotides. Phys Rev. E. 1994; 49(2): 1685-1689.

[18] Lee JM, Kim DJ, Kim IY, Park KS, Kim SI. Nonlinear-analysis of human sleep EEG using detrended fluctuation analysis. Med Eng and Phys. 2004; 26(9): 773-776.

[19] Ignaccolo M, Latka M, Jernajczyk W, Grigolini P, West BJ. Dynamics of electroencephalogram entropy and pitfalls of scaling detection. Phys Rev E. 2010; 81: 031909.

[20] Penzel T, Kantelhardt JW, Grote L, Peter JH, Bunde A. Comparison of detrended fluctuation analysis and spectral analysis for heart rate variability in sleep and sleep apnea. IEEE Trans BioMed Eng. 2003; 50(10): 1143-1151.

[21] American national standard (ANSI/AAMI EC13:2002). Cardiac monitors, heart rate meters, and alarms [Internet]. Arlington, VA: Association for the Advancement of Medical Instrumentation. 2002; [Updated 2002 May 6; Cited 2014 Jul 11]. Available from: http://www.pbme.ir/wp-content/uploads/2014/12/ec13.pdf.

[22] PhysioNet. Detrended Fluctuation Analysis (DFA) [Internet]. PhysioNet; [Updated 2012 Aug 15; Cited 2014 Jul 11]. Available from: http://www.physionet.org/physiotools/dfa/\#software-for-dfa. 